\title{
CASTELNUOVO-MUMFORD REGULARITY AND CLASSICAL METHOD OF CASTELNUOVO
}

\author{
ChiKashi MiYazaKi
}

\begin{abstract}
This paper investigates the Castelnuovo-Mumford regularity of generic hyperplane section of projective curve. The classical method of Castelnuovo plays an important role in order to study the extremal examples for the bounds for the CastelnuovoMumford regularity.
\end{abstract}

\section{Introduction}

This paper investigates the Castelnuovo-Mumford regularity of a generic hyperplane section of a projective curve. Let $T=k\left[y_{0}, \ldots, y_{N+1}\right]$ be the polynomial ring over an algebraically closed field $k$. Then we put $\mathbf{P}_{k}^{N+1}=$ $\operatorname{Proj}(T)$. Let $C$ be an irreducible reduced nondegenerate projective curve in $\mathbf{P}_{k}^{N+1}$, that is, the defining ideal $I_{C}$ is generated by elements of degree $\geq 2$ in $T$ and $T / I_{C}$ is an integral domain of dimension 2. Let $X$ be a generic hyperplane section of $C$, that is, $X=C \cap H$, where $H$ is a generic hyperplane of $\mathbf{P}_{k}^{N+1}$. So, $X$ is a zero-dimensional subscheme of $\mathbf{P}_{k}^{N}=\operatorname{Proj}(S)$, where $S$ is the polynomial ring $k\left[x_{0}, \ldots, x_{N}\right]$. Let $I$ be the defining ideal of $X$ and $R$ be the coordinate ring of $X$, that is, $R=S / I$. For a coherent sheaf $\mathscr{F}$ on $\mathbf{P}_{k}^{N}$ and an integer $m \in \mathbf{Z}, \mathscr{F}$ is said to be $m$-regular if $\mathrm{H}^{i}\left(\mathbf{P}_{k}^{N}, \mathscr{F}(m-i)\right)=0$ for all $i \geq 1$. For a projective scheme $Y \subseteq \mathbf{P}_{k}^{N}, Y$ is said to be $m$-regular if the ideal sheaf $\mathscr{I}_{Y}$ is $m$ regular. So, in this case, $X$ is $m$-regular if and only if $\mathrm{H}^{1}\left(\mathbf{P}_{k}^{N}, \mathscr{I}_{X}(m-1)\right)=0$, where $\mathscr{I}_{X}$ is the ideal sheaf of $X$. The Castelnuovo-Mumford regularity of $X \subseteq \mathbf{P}_{k}^{N}$ is the least such integer $m$ and is denoted by $\operatorname{reg}(X)$. It is well-known that $X$ is $m$-regular if and only if for every $p \geq 0$ the minimal generators of the $p$ th syzygy module of the defining ideal $I$ of $X \subseteq \mathbf{P}_{k}^{N}$ occur in degree $\leq m+p$. In this sense, it is important to study upper bounds on the Castelnuovo-Mumford regularity for projective schemes in order to describe the minimal free resolutions of the defining ideals. The following result (1.1) is a starting point of our

1991 Mathematics Subject Classification. Primary 14N05; Secondary 13D02, 14H99.

Partially supported by Grant-in-Aid for Scientific Research (C) (17540035), Ministry of Education, Culture, Sports, Science and Technology, Japan.

Received June 30, 2004; revised December 7, 2005. 
research on the Castelnuovo-Mumford regularity for generic hyperplane sections of projective curves. Throughout this paper, for a rational number $n \in \mathbf{Q},\lceil n\rceil$ denotes the smallest integer which is not less than $n$.

Proposition 1.1. Let $X \subseteq \mathbf{P}_{k}^{N}$ be a generic hyperplane section of a nondegenerate projective curve. Then we have $\operatorname{reg}(X) \leq\lceil(\operatorname{deg}(X)-1) / \operatorname{codim}(X)\rceil+1$.

Before describing a sketch of the proof of (1.1), we explain the terms "uniform position", "linear general position" and "linear semi-uniform position" for zero-dimensional schemes. Let $X \subseteq \mathbf{P}_{k}^{N}$ a reduced zero-dimensional scheme such that $X$ spans $\mathbf{P}_{k}^{N}$ as $k$-vector space. Then $X$ is said to be in uniform position if $\mathrm{H}_{Z}(t)=\max \left\{\operatorname{deg}(Z), \mathrm{H}_{X}(t)\right\}$ for all $t$, for any subscheme $Z$ of $X$, where $\mathrm{H}_{Z}$ and $\mathrm{H}_{X}$ denote the Hilbert function of $Z$ and $X$ respectively. This condition is equivalent to saying that, for any subschemes $Z_{1}$ and $Z_{2}$ of $X$ with $\operatorname{deg}\left(Z_{1}\right)=\operatorname{deg}\left(Z_{2}\right), \quad h^{0}\left(\mathbf{P}_{k}^{N}, \mathscr{I}_{Z_{1}}(\ell)\right)=\mathrm{h}^{0}\left(\mathbf{P}_{k}^{N}, \mathscr{I}_{Z_{2}}(\ell)\right)$ for all integers $\ell \in \mathbf{Z}$. A reduced zero-dimensional scheme $X$ is said to be in linear semi-uniform position if there are integers $v(i, X)$, simply written as $v(i), 0 \leq i \leq N$ such that every $i$ plane $L$ in $\mathbf{P}_{k}^{N}$ spanned by linearly independent $i+1$ points of $X$ contains exactly $v(i)$ points of $X$. We say $X$ is in linear general position if $v(i)=i+1$ for all $i \geq 1$. Further, we note that "uniform position" implies "linear general position", see $[7,(4.3)]$.

Remark 1.2. A generic hyperplane section of a nondegenerate projective curve is in linear semi-uniform position, see [2], and in uniform position if $\operatorname{char}(k)=0$, see [1].

The property of $h$-vectors for zero-dimensional scheme in linear semi-uniform position yields the proof of Proposition 1.1. Now we sketch the proof for the readers' convenience.

Sketch of the proof of Proposition 1.1. Let $R$ be the coordinate ring of a zero-dimensional scheme $X \subseteq \mathbf{P}_{k}^{N}$. Let $\underline{h}=\underline{h}(X)=\left(h_{0}, \ldots, h_{s}\right)$ be the $h$-vector of $X \subseteq \mathbf{P}_{k}^{N}$, where $h_{i}=\operatorname{dim}_{k}[R]_{i}-\operatorname{dim}_{k}[R]_{i-1}$ and $s$ is the largest integer such that $h_{s} \neq 0$. Note that $s=\operatorname{reg}(X)-1$. Since $X$ is in linear semi-uniform position, we have $h_{1}+\cdots+h_{i} \geq i h_{i}$ for all $i=1, \ldots, s-1$, that is, $\mathrm{H}_{X}(t) \geq$ $\min \{\operatorname{deg}(X), t N+1\}$ by [2]. Since $\operatorname{deg}(X)=h_{0}+\cdots+h_{s}$ and $\operatorname{codim}(X)=h_{1}=$ $N$, we obtain $\lceil(\operatorname{deg}(X)-1) / \operatorname{codim}(X)\rceil=\left\lceil\left(h_{1}+\cdots+h_{s}\right) / h_{1}\right\rceil \geq s$. Hence the assertion is proved.

Remark 1.3. For a nondegenarate reduced zero-dimensional scheme $X \subseteq \mathbf{P}_{k}^{N}$ in linear semi-uniform position, $\operatorname{reg}(X) \leq\lceil(\operatorname{deg}(X)-1) / \operatorname{codim}(X)\rceil+1$ holds from the proof of (1.1). On the other hand, when $X$ is in linear general position, the classical method of Castelnuovo gives the inequality, see [1, page 115] or [4, page 95]. 
We will study extremal cases for regularity bounds in Proposition 1.1. Now we state the main theorem, which extends the results of $[4,(2.4)]$.

THEOREM 1.4. Let $X \subseteq \mathbf{P}_{k}^{N}$ be a generic hyperplane section of a nondegenerate projective curve $C$ in $\mathbf{P}_{k}^{N+1}$ for $N \geq 3$. Assume that $\operatorname{deg}(X) \geq N^{2}+$ $2 N+2$. If the equality $\operatorname{reg}(X)=\lceil(\operatorname{deg}(X)-1) / \operatorname{codim}(X)\rceil+1$ holds, then $X$ is contained in a rational normal curve in $\mathbf{P}_{k}^{N}$.

We remark here the hypothesis $\operatorname{deg}(X) \geq N^{2}+2 N+2$ is indispensable because of an example of a $(2,2,4)$ complete intersection in $\mathbf{P}_{k}^{3}$, see [9].

For $N=1$, the curve $C$ is defined by one equation of degree $d$, and we easily have $\operatorname{reg}(X)=d$. Then we have an equality $\operatorname{reg}(X)=\lceil(\operatorname{deg}(X)-1) / \operatorname{codim}(X)\rceil$ +1 . For $N \geq 2$, the corresponding result is obtained by the Hilbert-Burch matrix, see [5].

Proposition 1.5 (See [9, (2.6)]). Let $X \subseteq \mathbf{P}_{k}^{N}$ be a generic hyperplane section of a nondegenerate projective curve. Assume that $X$ is in uniform position and $\operatorname{deg}(X) \geq N^{2}+2 N+2$. If the equality $\operatorname{reg}(X)=\lceil(\operatorname{deg}(X)-1) / N\rceil+1$ holds, then $X$ is contained in a rational normal curve in $\mathbf{P}_{k}^{N}$.

From (1.5) we focus on the case that $X$ is not in uniform position. So, $k$ is assumed to be a field of positive characteristic. Theorem 1.4 is reduced to Theorem 1.6.

THEOREM 1.6. Let $X \subseteq \mathbf{P}_{k}^{N}$ be a generic hyperplane section of a nondegenerate projective curve for $N \geq 3$. Assume that $X$ is not in uniform position and $\operatorname{deg}(X) \geq N^{2}+2 N+2$. Then we have $\operatorname{reg}(X) \leq\lceil(\operatorname{deg}(X)-1) / N\rceil$.

What we have to prove is that $\mathrm{H}^{0}\left(\mathcal{O}_{\mathbf{P}_{k}^{N}}(t)\right) \rightarrow \mathrm{H}^{0}\left(\mathcal{O}_{X}(t)\right)$ is surjective, that is, $\mathrm{H}^{1}\left(\mathscr{I}_{X}(t)\right)=0$, where $t=\lceil(\operatorname{deg}(X)-1) / N\rceil-1$.

Lemma 1.7. Under the condition of (1.6), let $t=\lceil(\operatorname{deg}(X)-1) / N\rceil-1$. For any fixed point $P \in X$, there exists a (possibly reducible) hypersurface $F$ of degree $t$ in $\mathbf{P}_{k}^{N}$ such that $X \cap F=X \backslash\{P\}$.

Section 2 is devoted to the proof of Lemma 1.7, which is a consequence of Theorem 2.2. In Section 3, we study the configuration of the zero-dimensional scheme of $\mathbf{P}_{k}^{2}$ in linear semi-uniform position having the extremal bound for the regularity. Throughout this paper, the classical method of Castelnuovo plays an important role in order to describe the Castelnuovo-Mumford regularity for the zero-dimensional scheme.

\section{Curve in $\mathbf{P}^{n}(n \geq 4)$}

In this section, we investigate extremal bounds on the Castelnuovo-Mumford regularity by the classical method in order to prove Lemma 1.7 for a generic 
hyperplane section of a nondegenerate projective curve $C$ in $\mathbf{P}^{n}(n \geq 4)$. Now we begin with a useful result of Rathmann [10] by describing a relation between the monodromy group of the projective curve and the configuration of the generic hyperplane section of the curve. For a generic hyperplane section $X \subseteq \mathbf{P}_{k}^{N}$ of a nondegenerate projective curve $C \subseteq \mathbf{P}_{k}^{N+1}$, let $M \subseteq C \times\left(\mathbf{P}_{k}^{N+1}\right)^{*}$ be the incidence correspondence parametrizing the pairs $(x, H) \in M$ such that $x$ is contained in $H$. Since $M$ is generically étale finite over $P=\left(\mathbf{P}_{k}^{N+1}\right)^{*}$ via the second projection, the function field $K(M)$ of $M$ is separable finite over $K(P)$. For a splitting field $Q$ for $K(M) / K(P)$, we take the Galois group $G_{C}=\mathrm{Gal}(Q / K(P))$. Then $G_{C}$ is a subgroup of the full symmetric group $S_{d}$ and is called the monodromy group of $C \subseteq \mathbf{P}_{k}^{N}$, where $d=\operatorname{deg}(C)$. The following classification correponds with the transitivity of the monodromy group of the projective curve.

Proposition 2.1 (See $[10,(2.5)])$. Let $X \subseteq \mathbf{P}^{N}$ be a generic hyperplane section of a nondegenerate projective curve $C \subseteq \mathbf{P}^{N+1}$ for $N \geq 3$. Let $G_{C}$ be the monodromy group of $C$. If $X$ is not in uniform position, then either of the following holds:

(a) $v(1)=3$, and $G_{C}$ is exactly 2-transitive.

(b) $v(1)=2, v(2) \geq 4$, and $G_{C}$ is exactly 3-transitive.

(c) $\operatorname{deg}(C)=11,12,23$ or 24 , and $G_{C}$ is the Mathieu group $M_{11}, M_{12}$, $M_{23}, M_{24}$ respectively. Moreover $M_{11}$ and $M_{23}$ are exactly 4-transitive and $N \geq 4$ for the case. Also, $M_{12}$ and $M_{24}$ are exactly 5-transitive and $N \geq 5$ for the case.

Now we state the main theorem of this section.

THEOREM 2.2. Let $X \subseteq \mathbf{P}_{k}^{N}$ be a generic hyperplane section of a nondegenerate projective curve $C$ of degree $d$ in $\mathbf{P}_{k}^{N+1}$ for $N \geq 3$. Assume that $X$ is not in uniform position and that $d \geq N^{2}+2 N+2$. For any fixed point $P \in X$, there exists a (possibly reducible) hypersurface $F$ of degree $t$ in $\mathbf{P}_{k}^{N}$ such that $X \cap F=X \backslash\{P\}$, where $t=\lceil(d-1) / N\rceil-1$. In other words, $\operatorname{reg}(X) \leq$ $\lceil(d-1) / N\rceil$.

According to the classification (2.1) we will prove (2.2) in each case separately. As for the case (c) in (2.1), since $N \geq 4$ for $\operatorname{deg}(C)=11,23$ and $N \geq 5$ for $\operatorname{deg}(C)=12,24$, we see $N^{2}+2 N+2 \geq 26$. Hence there is no curves with degree $11,12,23$ or 24 satisfying the condition. Thus the case (c) in (2.1) is proved. The rest of the proof of Theorem 2.2 is divided by (2.3), (2.4) and (2.5).

First we study the case (a) in (2.1).

Lemma 2.3. Under the condition of (2.2), assume that $v(1)=3$, that is, $G_{C}$ is exactly 2-transitive. For any fixed point $P \in X$, there exists (possibly reducible) hypersurface $F$ of degree $t$ in $\mathbf{P}_{k}^{N}$ such that $X \cap F=X \backslash\{P\}$, where $t=$ $\lceil(d-1) / N\rceil-1$. 
Proof. For the case $N \geq 4$, the classical method have given the proof of the assertion in $[4,(2.2)]$. So we will prove for the case $N=3$ by the classical method. Since $v(1)=3$, we have $v(2) \geq 7$ and put $v=v(2)$. For a point $P$ of $X$, we fix 2 points $Q_{1}$ and $Q_{2}$ in $X \backslash\{P\}$. Then we take different 2-planes $L_{1}, \ldots, L_{a}$ containing the line $\ell=\ell\left(Q_{1}, Q_{2}\right)$ spanned by $Q_{1}$ and $Q_{2}$ such that the union $\bigcup_{j=1}^{a} L_{j}$ covers $X$. We remark that $a \geq 3$. Since each 2-plane contains exactly $v$ points of $X$ and the line $\ell$ contains exactly 3 points of $X$, we see $d=a(v-3)+3$. We may assume that $P$ is contained in $L_{a}$. Let $b=$ $\lceil(v-3) / 2\rceil$. Since $\left(X \cap L_{a}\right) \backslash\left\{P, Q_{1}, Q_{2}\right\}$ consists of exactly $v-3$ points, there are 2-planes $L_{1}^{\prime}, \ldots, L_{b}^{\prime}$ such that $P \notin L_{i}^{\prime}$ for $i=1, \ldots, b$ and the union $\bigcup_{j=1}^{b} L_{j}^{\prime}$ of 2-planes covers $\left(X \cap L_{a}\right) \backslash\left\{P, Q_{1}, Q_{2}\right\}$. By taking $F=\left(\bigcup_{i=1}^{a-1} L_{i}\right) \cup\left(\bigcup_{j=1}^{b} L_{j}^{\prime}\right)$, we have $(X \cap F)=X \backslash\{P\}$ and the degree of the union $F$ of 2 -planes is $a+b-1$. Thus we have only to show that $(a-1)+\lceil(v-3) / 2\rceil \leq\lceil((a v-3 a+3)-1) / 3\rceil$ -1 . The inequality $(a-1)+(v-3) / 2 \leq(a v-3 a+2) / 3-1$ is equivalent to saying that $(2 a-3)(v-6) \geq 5$, which is easily shown for $v \geq 7$ and $a \geq 4$. Moreover, the case $v=7$ and $a=3$ satisfies $(a-1)+\lceil(v-3) / 2\rceil=\lceil(a v-3 a+$ $2) / 3\rceil-1$. Hence the assertion is proved.

Next we study for the case (b) in (2.1) and $N=3$.

LEMMA 2.4. Under the condition of (2.2), assume that $v(1)=2, v(2) \geq 4$ and $N=3$, and $G_{C}$ is exactly 3-transitive. For any fixed point $P \in X$, there exists a union $F$ of $t$ hyperplanes $L_{1}, \ldots, L_{t}$ in $\mathbf{P}_{k}^{4}$ such that $X \cap F=X \backslash\{P\}$, where $t=\lceil(d-1) / 3\rceil-1$.

Proof. Let us put $v=v(2) \geq 4$. For the case $v \geq 5$, the proof will be proceeded as in (2.3). For a point $P$ of $X$, we fix 2 points $Q_{1}$ and $Q_{2}$ in $X \backslash\{P\}$. Then we take different 2-planes $L_{1}, \ldots, L_{a}$ containing the line $\ell=$ $\ell\left(Q_{1}, Q_{2}\right)$ such that the union $\bigcup_{j=1}^{a} L_{j} \operatorname{covers} X$. In this case, we see that $a \geq 2$ and $d=a(v-2)+2$. We may assume that $P$ is contained in $L_{a}$. Let $b=$ $\lceil(v-3) / 2\rceil$. Then there are 2-planes $L_{1}^{\prime}, \ldots, L_{b}^{\prime}$ such that $P \notin L_{i}^{\prime}$ for $i=1, \ldots, b$ and the union $\bigcup_{j=1}^{b} L_{j}^{\prime}$ of 2-planes covers $\left(X \cap L_{a}\right) \backslash\left\{P, Q_{1}, Q_{2}\right\}$. By taking $F=\left(\bigcup_{i=1}^{a-1} L_{i}\right) \cup\left(\bigcup_{j=1}^{b} L_{j}^{\prime}\right)$, we have $(X \cap F)=X \backslash\{P\}$ and $\operatorname{deg}(F)=a+b-1$. Thus the assertion is reduced to showing that $(a-1)+\lceil(v-3) / 2\rceil \leq\lceil(a v-2 a+$ $1) / 3\rceil-1$. The inequality $(a-1)+(v-3) / 2 \leq(a v-2 a+1) / 3-1$ is equivalent to saying that $(2 a-3)(v-5) \geq 4$, which is easily shown for all $v \geq 6$ and $a \geq 2$ satisfying $a v-2 a+2 \geq 17$. For the case $v=5$, we see that $(a-1)+1=$ $\lceil(3 a+1) / 3\rceil-1$, which gives the assertion.

Next we consider the case $v=4$. As the notations above, $d=2 a+2$ and $a \geq 8$. For a point $P$ of $X$, we will take hyperplanes $L_{1}, \ldots, L_{\ell_{1}}$ inductively for some integer $\ell_{1}$ such that $P \notin L_{i}$ and $X \cap L_{i} \backslash \bigcup_{j=1}^{i-1} L_{j}$ contains exactly 4 points of $X \backslash\{P\}$. Indeed, let $Y_{i}=X \cap\left(L_{1} \cup \cdots \cup L_{i}\right)$ and $Z_{i}=X \backslash\left(\{P\} \cup Y_{i}\right)$. Let us take $Q_{1}$ and $Q_{2}$ in $Z_{i}$. Then there exists a point $Q_{3}$ in of $Z_{i} \backslash\left\{Q_{1}, Q_{2}\right\}$ such that the 2-plane $L_{i+1}=L\left(Q_{1}, Q_{2}, Q_{3}\right)$ spanned by $Q_{1}, Q_{2}$ and $Q_{3}$ does not contain 
any point of $Y_{i} \cup\{P\}$ if $\operatorname{card}\left(Z_{i} \backslash\left\{Q_{1}, Q_{2}\right\}\right)>\operatorname{card}\left(Y_{i} \cup\{P\}\right)$. Thus the 2-plane $\left(X \cap L_{i+1}\right) \backslash Y_{i}$ contains exactly 4 points. Therefore we have constructed a union of hyperplanes $G_{\ell_{1}}=L_{1} \cup \cdots \cup L_{\ell_{1}}$ such that $G_{\ell_{1}}$ contains at least $a+1$ points of $X$ and does not contain $P$. Hence we have $\ell_{1}=\lceil(a+1) / 4\rceil$ and $\operatorname{card}\left(X \cap G_{\ell_{1}}\right)$ $=4 \ell_{1}$.

Moreover, we will inductively construct hyperplanes $L_{\ell_{1}+1}, \ldots, L_{\ell_{1}+\ell_{2}}$ for some integer $\ell_{2}$ satisfying $X \backslash\{P\}=X \cap\left(L_{1} \cup \cdots \cup L_{\ell_{1}+\ell_{2}}\right)$. Let $G_{i}=L_{1} \cup \cdots \cup L_{i}$. If $\operatorname{card}\left(X \backslash\left(\{P\} \cup G_{i}\right)\right)>6$, we take a hyperplane $L_{i+1}$ such that $L_{i+1}$ contains at least 3 points of $X \backslash G_{i}$ and does not contain $P$. For the case $\operatorname{card}(X \backslash$ $\left.\left(\{P\} \cup G_{i}\right)\right)=4,5,6$, we can take hyperplanes which covers the remaining points and does not contain $P$. Indeed we may assume that $\operatorname{card}\left(X \backslash\left(\{P\} \cup G_{i}\right)\right)=6$. Let $X \backslash\left(\{P\} \cup G_{i}\right)=\left\{Q_{1}, \ldots, Q_{6}\right\}$. Let us take hyperplanes $M_{1}=L\left(Q_{1}, Q_{2}, P\right)$ and $M_{2}=L\left(Q_{3}, Q_{4}, P\right)$. If $M_{1} \cap M_{2} \cap\left\{Q_{1}, \ldots, Q_{6}\right\}=\phi$, then we may assume that $Q_{5} \notin M_{1}$ and $Q_{6} \notin M_{2}$. So, we see that $P \notin L\left(Q_{1}, Q_{2}, Q_{5}\right)$ and $P \notin L\left(Q_{3}\right.$, $\left.Q_{4}, Q_{6}\right)$. If $M_{1} \cap M_{2} \cap\left\{Q_{1}, \ldots, Q_{6}\right\} \neq \phi$, say the intersection is a point $Q_{5}$, then $P \notin L\left(Q_{1}, Q_{5}, Q_{6}\right)$ and $P \notin L\left(Q_{2}, Q_{3}, Q_{4}\right)$. Thus we have that $\ell_{2} \leq\lceil(2 a+2-$ $\left.\left.4 \ell_{1}\right) / 3\right\rceil \leq\lceil(a+1) / 3\rceil$. Hence the proof is reduced to showing that $\ell_{1}+\ell_{2} \leq$ $\lceil(d-1) / 3\rceil-1$, namely, $\lceil(a+1) / 4\rceil+\lceil(2 a+2-4\lceil(a+1) / 4\rceil) / 3\rceil \leq\lceil(2 a+1) / 3\rceil$ -1 for $a \geq 8$, which is easily verified. Hence the assertion is proved.

Finally we study the case (b) in (2.1) and $N \geq 4$. We will show the assertion without using [3], although the proof proceeds as in [4] by the classical method.

LEMMA 2.5. Let $X \subseteq \mathbf{P}_{k}^{N}$ be a zero-dimensional scheme of degree $d \geq$ $N^{2}+2 N+2$ in linear semi-uniform position. Assume that $v(1)=2, v(2) \geq 4$ and $N \geq 4$. For any fixed point $P \in X$, there exists a (possibly reducible) hypersurface $F$ of degree $t$ in $\mathbf{P}_{k}^{N}$ such that $X \cap F=X \backslash\{P\}$, where $t=\lceil(d-1) / N\rceil-1$. In other words, $\operatorname{reg}(X) \leq\lceil(d-1) / N\rceil$.

Proof. First, we will show that $v(i+1) \geq 2 v(i)$ for $i \geq 2$. Indeed, let us take an $i$-plane $G$ spanned by linearly independent $i+1$ points of $X$, and take a point $A_{1} \in X \cap G$ and a point $A_{2} \in X \backslash(X \cap G)$. Then we put $X \cap G=\left\{A_{1}\right\} \cup$ $\left\{B_{1}, \ldots, B_{v(i)-1}\right\}$. For any point $B_{j} \in(X \cap G) \backslash\left\{A_{1}\right\}$, the 2-plane $H=H\left(A_{1}, A_{2}\right.$, $\left.B_{j}\right)$ spanned by $A_{1}, A_{2}, B_{j}$ contains at least one point $C_{j}$ in $(X \cap H) \backslash((X \cap G) \cup$ $\left\{A_{2}\right\}$ ) for all $j$, because $v(2) \geq 4$ and $v(1)=2$. Note that $C_{j} \neq C_{j^{\prime}}$ for $j \neq j^{\prime}$. So, the $(i+1)$-plane spanned by $G$ and $A_{2}$ contains $A_{1}, A_{2}, B_{1}, \ldots, B_{v(i)-1}, C_{1}, \ldots$, $C_{v(i)-1}$. Thus we have $v(i+1) \geq 2 v(i)$ for $i \geq 2$. Let $v=v(N-2)$ and $w=$ $v(N-1)$. Since $v(2) \geq 4$, we see that $v(i) \geq 2^{i}$ for $i \geq 2$ and $w \geq 2 v \geq 2^{N-1}$.

Now, we fix linearly independent $N-1$ points $Q_{1}, \ldots, Q_{N-1}$ of $X \backslash\{P\}$ such that the $(N-2)$-plane $L$ spanned by $Q_{1}, \ldots, Q_{N-1}$ does not contain the point $P$. Thus there are different hyperplanes $L_{1}, \ldots, L_{a}$ containing $L$ such that the union $\bigcup_{j=1}^{a} L_{j}$ covers $X$. So, we easily have that $d=a(w-v)+v$ and $a \geq 2$. Let us prove that $a \geq v(2)-1$. Indeed, let us take points $Q_{1}^{\prime} \in L_{1} \backslash L$ and $Q_{2}^{\prime} \in L_{2} \backslash L$. 
Then the 2-plane $L^{\prime}=L\left(Q_{1}, Q_{1}^{\prime}, Q_{2}^{\prime}\right)$ contains $v(2)$ points of $X$, say, $X \cap L^{\prime}=$ $\left\{Q_{1}, Q_{1}^{\prime}, Q_{2}^{\prime}, Q_{3}^{\prime}, \ldots, Q_{v(2)-1}^{\prime}\right\}$. Since $v(1)=2$, the 2-plane $L_{i(j)}$ containing $Q_{j}$ are different from each other for $j=1, \ldots, v(2)-1$. Hence we have $a \geq v(2)-1$.

We will prove the result by the induction on $N$. Let $N \geq 4$. From the notation above, we may assume that $P$ is contained in $L_{a}$. Since $X \cap L_{a}$ is also in linear semi-uniform position in $L_{a}\left(\cong \mathbf{P}_{k}^{N-1}\right)$, by (1.3) there is a (possibly reducible) hypersurface $F^{\prime}$ in $\mathbf{P}_{k}^{N}$ of degree $\lceil(w-1) /(N-1)\rceil$ such that $P \notin F^{\prime}$ and $F^{\prime}$ contains $\left(X \cap L_{a}\right) \backslash\{P\}$. Thus the union $F=\left(\bigcup_{j=1}^{a-1} L_{j}\right) \cup F^{\prime}$ covers $X \backslash\{P\}$ and $P \notin F$. Hence it suffices to prove that $a-1+\lceil(w-1) /(N-1)\rceil \leq$ $\lceil(d-1) / N\rceil-1$, that is, $a+\lceil(w-1) /(N-1)\rceil \leq\lceil(a(w-v)+v-1) / N\rceil$. For $N \geq 5$, let us consider an inequality $a+(w-1) /(N-1) \leq(a(w-v)+v-1) / N$, which is equivalent to $a N^{2}-a N-1 \leq(a N-a-N) w-(N-1)(a-1) v$. Since $a N-a-N \geq 0$, we see that $(a N-a-N) w-(N-1)(a-1) v \geq 2(a N-a-N) v$ $-(N-1)(a-1) v=(a N-N-a-1) v \geq a N^{2}-a N+1$ for $N \geq 5$ except for $(N, a, v)=(5,3,8)$ or $(5,3,9)$, because $v \geq 2^{N-2}$. For the case, since $d=3 w-$ $2 v \geq 37$ and $w \geq 2 v$, we have $3+\lceil(w-1) / 4\rceil \leq\lceil(3 w-2 v-1) / 5\rceil$. Therefore we have the desired inequality for $N \geq 5$.

Next let $N=4$. Let us assume that $(v, w) \neq(4,8)$ as notation above. Then we easily have an inequality $a+\lceil(w-1) / 3\rceil \leq\lceil(a(w-v)+v-1) / 4\rceil$ except for $(a, v, w)=(4,5,11)$ or $(5,4,9)$. For the case $(a, v, w)=(4,5,11)$, the union of hyperplanes $L_{1} \cup L_{2} \cup L_{3}$ contains exactly $23(=11+6+6)$ points of $X$ and does not contain $P$. Since $\operatorname{card}(X \backslash\{P\})=28$, there exists a union of 3 hyperplanes which covers the remaining 5 points of $X \backslash\{P\}$ and does not contain $P$. Thus we have done. For the case $(a, v, w)=(5,4,9)$, the union of hyperplanes $L_{1} \cup \cdots \cup L_{4}$ contains exactly $23(=9+5+5+5)$ points of $X$ and does not contain $P$. Since card $(X \backslash\{P\})=28$, there exists a union of 2 hyperplanes which covers the remaining 5 points of $X \backslash\{P\}$ and does not contain $P$. Thus we have done.

Finally let $N=4$ and $(v, w)=(4,8)$ as notations above. Note that $\operatorname{card}(X)=d=4 a+4$ and $a \geq 6$. Let us take a hyperplane $H_{1}$ which contains exactly 8 points of $X \backslash\{P\}$. Then we will take hyperplanes $H_{2}, \ldots, H_{\ell_{1}}$ inductively for some integer $\ell_{1}$ such that $P \notin H_{i}$ and $\left(X \cap H_{i}\right) \backslash \bigcup_{j=1}^{i-1} H_{j}$ contains at least 7 points. Indeed, let $Y_{i}=X \cap\left(H_{1} \cup \cdots \cup H_{i}\right)$ and $Z_{i}=X \backslash\left(\{P\} \cup Y_{i}\right)$. Let us take $Q_{1}$ and $Q_{2}$ in $Z_{i}$. Then we take a point $Q_{3}$ in of $Z_{i} \backslash\left\{Q_{1}, Q_{2}\right\}$ such that the 2-plane $L=L\left(Q_{1}, Q_{2}, Q_{3}\right)$ does not contain any point of $Y_{i} \cup\{P\}$ if $\operatorname{card}\left(Z_{i} \backslash\left\{Q_{1}, Q_{2}\right\}\right)>\operatorname{card}\left(Y_{i} \cup\{P\}\right)$. Thus the 2-plane $L$ contains exactly 4 points $Q_{1}, \ldots, Q_{4}$ of $Z_{i}$. Then there exists a point $Q_{5}$ of $Z_{i} \backslash L$ such that the hyperplane $M$ spanned by $Q_{5}$ and $L$ contains at least two other points $Q_{6}, Q_{7}$ of $Z_{i} \backslash L$ and does not contain $P$ if $\operatorname{card}\left(Z_{i} \backslash L\right)>\operatorname{card}\left(Y_{i}\right)+3$. Thus we can take $H_{i+1}=M$ which satisfies the condition. Therefore we have constructed a union of hyperplanes $G_{\ell_{1}}=H_{1} \cup \cdots \cup H_{\ell_{1}}$ such that $G_{\ell_{1}}$ contains at least $(d-8) / 2=$ $2 a-2$ points of $X$ and does not contain $P$, and $\ell_{1} \leq\lceil(2 a-3) / 7\rceil$. Moreover, we will inductively construct hyperplanes $H_{\ell_{1}+1}, \ldots, H_{\ell_{1}+\ell_{2}}$ for some integer $\ell_{2}$ satisfying $X \backslash\{P\}=X \cap\left(H_{1} \cup \cdots \cup H_{\ell_{1}+\ell_{2}}\right)$. Let $G_{i}=H_{1} \cup \cdots \cup H_{i}$. If $\operatorname{card}(X \backslash$ 
$\left.\left(\{P\} \cup G_{i}\right)\right)>6$, we take a hyperplane $H_{i+1}$ such that $H_{i+1}$ contains at least 4 points of $X \backslash G_{i}$ and does not contain $P$. For the case $\operatorname{card}\left(X \backslash\left(\{P\} \cup G_{i}\right)\right)=$ $3,4,5,6$, we can take hyperplanes which covers the remaining points and does not contain $P$, see the proof of (2.4), and for the case $\operatorname{card}\left(X \backslash\left(\{P\} \cup G_{i}\right)\right)=1,2$, we can take one hyperplanes as desired. Thus we see that $\ell_{2} \leq\lceil(d-(2 a-2)+$ $2) / 4\rceil=\lceil(a+4) / 4\rceil$. Hence the proof is reduced to showing that $\ell_{1}+\ell_{2} \leq$ $\lceil(d-1) / 4\rceil-1$, namely, $\lceil(2 a-3) / 7\rceil+\lceil(a+4) / 4\rceil \leq a$ for $a \geq 6$, which is easily verified. Hence we have proved the case $N=4$.

\section{Curve in $\mathbf{P}^{3}$ and zero-dimensional scheme in $\mathbf{P}^{2}$}

In this section, we investigate extremal bounds on the Castelnuovo-Mumford regularity by the classical method of Castelnuovo for nondegenerate reduced zerodimensional scheme of $\mathbf{P}_{k}^{2}$ in linear semi-uniform position. If a generic hyperplane section $X$ of a space curve is in uniform position, there is an arithmetically Cohen-Macaulay smooth curve $C^{\prime} \subseteq \mathbf{P}_{k}^{3}$ such that $X=C^{\prime} \cap H$. This gives detailed information of a free resolution of the defining ideal $I_{X}$ over $k\left[x_{0}, x_{1}, x_{2}\right]$ by the Hilbert-Burch matrix, see [6]. Instead, we will go ahead with a classical method, since a generic hyperplane section of a space curve is in linear semiuniform position.

THEOREM 3.1. Let $X$ be a nondegenerate reduced zero-dimensional scheme of $\mathbf{P}_{k}^{2}$ of degree $d . \quad$ Assume that $X$ is in linear semi-uniform position and $d \geq 10$. If the equality $\operatorname{reg}(X)=\lceil(d-1) / 2\rceil+1$ holds, then either (i) $X$ is contained in a conic, or (ii) $X=\{P\} \cup Y$, where $Y$ is contained in a conic.

Example 1. Let $X$ be a reduced zero-dimensional scheme in a conic of $\mathbf{P}_{k}^{2}$ of degree $d \geq 3$. Then we easily have $\operatorname{reg}(X)=\lceil(d-1) / 2\rceil+1$, and $\underline{h}(X)=$ $(1,2, \ldots, 2)$ or $(1,2, \ldots, 2,1)$.

Example 2. Let $P$ be a point in $\mathbf{P}_{k}^{2}$ and $Y$ be a reduced zero-dimensional scheme in a conic of $\mathbf{P}_{k}^{2}$ with $P \notin Y$. Let $X=\{P\} \cup Y$. Assume that $X$ is in a linear general position and $d=\operatorname{deg}(X) \geq 10$. Then we easily have $\operatorname{reg}(X)=$ $\lceil(d-1) / 2\rceil+1$, and $\underline{h}(X)=(1,2,3,2, \ldots, 2)$ or $(1,2,3,2, \ldots, 2,1) . \quad$ In this case, $X$ is not of decreasing type, see [6, page 3139] for the definition of "decreasing type". According to $[5,(1.1)]$, the zero-dimensional scheme $X$ cannot be a generic hyperplane section of a space curve.

In case $X$ is in uniform position, the equality $\operatorname{reg}(X)=\lceil(d-1) / 2\rceil+1$ implies that $X$ is in a conic of $\mathbf{P}_{k}^{2}$ by $[9,(2.5)]$. Therefore, the proof of Theorem 3.1 is reduced to (3.2) and (3.3).

LeMmA 3.2. Under the condition of (3.1), assume that $X$ is not in general linear position. For any fixed point $P \in X$, there exists a union $F$ of $t$ lines $L_{1}, \ldots, L_{t}$ in $\mathbf{P}_{k}^{2}$ such that $X \cap F=X \backslash\{P\}$, where $t=\lceil(d-1) / 2\rceil-1$. 
Proof. Since $X$ is in linear semi-uniform position by [2], the line spanned by any two points of $X$ is contains exactly $v(1)$ points. Since $X$ is not in general linear position by assumption, we have $v=v(1) \geq 3$.

First we consider the case $v \geq 4$. For a point $P$ of $X$, let us take a point $Q$ in $X \backslash\{P\}$. Then we take different lines $\ell_{1}, \ldots, \ell_{a}$ through the point $Q$ such that the union $\bigcup_{j=1}^{a} \ell_{j}$ covers $X$. Each line contains the point $Q$ and the other $v-1$ points of $X$. Thus we see $d=a v-a+1$. We may assume that $P$ is contained in $\ell_{a}$. Then we take $L_{1}=\ell_{1}, \ldots, L_{a-1}=\ell_{a-1}$. Since $\left(X \cap \ell_{a}\right) \backslash\{P, Q\}$ consists of exactly $v-2$ points, we can easily take $v-2$ lines $L_{a}, \ldots, L_{a+v-3}$ such that the union $\bigcup_{j=a}^{a+v-3} L_{j}$ contains $\left(X \cap \ell_{a}\right) \backslash\{P, Q\}$ and $P \notin L_{i}$ for any $i$. Since $X \cap$ $\left(\bigcup_{j=1}^{a+v-3} L_{j}\right)=X \backslash\{P\}$, the assertion is reduced to showing that $a+v-3 \leq$ $\lceil(a v-(a-1)-1) / 2\rceil-1$ for $d=a v-(a-1) \geq 10$. Note that $a \geq v$. Indeed, let us take a point $P_{i}$ from $\left(X \cap \ell_{i}\right) \backslash\{P, Q\}$ for each $i=1,2$. Then we see $P_{1} \neq P_{2}$. Since the line $\ell\left(P_{1}, P_{2}\right)$ spanned by $P_{1}$ and $P_{2}$ contains $(v-2)$ points $P_{3}, \ldots, P_{v}$ in $X \backslash\left(\ell_{1} \cup \ell_{2}\right)$, the points $P_{3}, \ldots, P_{v}$ construct different lines $\ell_{3}^{\prime}=$ $\ell\left(P_{3}, Q\right), \ldots, \ell_{v}^{\prime}=\ell\left(P_{v}, Q\right)$ through $Q$. Since we have at least $v$ lines $\ell_{1}, \ell_{2}$, $\ell_{3}^{\prime}, \ldots, \ell_{v}^{\prime}$ through $Q$, we see $a \geq v$. The inequality $a+v-3 \leq a(v-1) / 2-1$ is equivalent to saying that $(a-2)(v-3) \geq 2$, which is easily shown for $a$ and $v$ with $a \geq v \geq 4$. Hence the assertion is proved.

Next we consider the case $v=3$. As the notations above, we see that $\operatorname{card}(X)=d=2 a+1$ and $a \geq 5$. For a point $P$ of $X$, we will take lines $L_{1}, \ldots, L_{a}$ inductively such that $L_{i} \backslash \bigcup_{j=1}^{i-1} L_{j}$ contains exactly 3 points of $X$ and $P \notin L_{i}$ for $i=1, \ldots, \ell_{1}$. Indeed, let $Y_{i}=X \cap\left(\bigcup_{j=1}^{i} L_{j}\right)$ and $Z_{i}=X \backslash\left(\{P\} \cup Y_{i}\right)$. Let us take a point $Q \in Z_{i}$. Then there exists a point $Q^{\prime} \in Z_{i}$ such that the line $L_{i+1}=\ell\left(Q, Q^{\prime}\right)$ spanned by $Q$ and $Q^{\prime}$ does not contain any points of $Y_{i} \cup\{P\}$ if $\operatorname{card}\left(Y_{i}\right)<\operatorname{card}\left(Z_{i}\right)-2$. Thus $\left(\bigcup_{j=1}^{\ell_{1}} L_{j}\right) \cap X$ consists of at least $a-1$ points and $\ell_{1}=\lceil(a-1) / 3\rceil$. Moreover, we will take lines $L_{\ell_{1}+1}, \ldots, L_{\ell_{2}}$ for some $\ell_{2}$ inductively such that $\left(X \cap L_{i}\right) \backslash \bigcup_{j=1}^{i-1} L_{j}$ contains at least 2 points for $i=\ell_{1}+$ $1, \ldots, \ell_{1}+\ell_{2}-1$ and $X \backslash\{P\}=X \cap\left(\bigcup_{j=1}^{\ell_{1}+\ell_{2}} L_{j}\right)$. Thus we see that $\ell_{2} \leq\lceil(d-$ $\left.\left.1-3 \ell_{1}\right) / 2\right\rceil$. Therefore the assertion is reduced to showing that $\lceil(a-1) / 3\rceil+$ $\lceil(2 a-3\lceil(a-1) / 3\rceil) / 2\rceil \leq a-1$, because $\lceil(d-1) / 2\rceil=a$. For $a=5,6,7,8$, the inequality holds. Since $2 a-3\lceil(a-1) / 3\rceil \leq a+1$, the proof of the inequality is reduced to showing that $\lceil(a-1) / 3\rceil+\lceil(a+1) / 2\rceil \leq a-1$, which is easily shown for $a \geq 9$. Hence the assertion is proved.

LEMmA 3.3. Under the condition of (3.1), assume that $X$ is in general linear position and not in uniform position. Assume that $X$ is not in the case (ii) in (3.1). For any fixed point $P \in X$, there exists a (possibly reducible) plane curve $F$ of degree $t$ in $\mathbf{P}_{k}^{2}$ such that $X \cap F=X \backslash\{P\}$, where $t=\lceil(d-1) / 2\rceil-1$.

Proof. Let $P$ be a point of $X$. Since $X$ is not in uniform position, $X$ is not contained in a conic of $\mathbf{P}_{k}^{2}$ by [8]. So there are a conic $C_{1}$ with $P \notin C_{1}$ containing at least 5 points of $X$. For the case $d=10$, there exsits a conic $C_{2}$ with $P \notin C_{2}$ containing $X \backslash\left(X \cap C_{1}\right)$, since the point $P$ and the 4 points 
$X \backslash\left(X \cap C_{1}\right)$ is not in a line. By taking $F=C_{1} \cup C_{2}$, we have $F \cap X=X \backslash\{P\}$ and $\operatorname{deg}(F)=4=\lceil(d-1) / 2\rceil-1$. Hence the assertion is proved.

So we may assume that $d \geq 11$. We have only to show that there are 2 conics $C_{1}$ and $C_{2}$ with $P \notin C_{1}$ and $P \notin C_{2}$ such that $\left(C_{1} \cup C_{2}\right) \cap X$ contain at least 10 points of $X$. Indeed, if there are such conics, the number of the remaining points of $X \backslash\left(\{P\} \cup\left(C_{1} \cup C_{2}\right)\right)$ is $m$, where $m=\lceil(d-11) / 2\rceil$. Since $X$ is in linear general position, there are $m$ lines $L_{1}, \ldots, L_{m}$, where $m=\lceil(d-11) / 2\rceil$, satisfying that $\left(\left(C_{1} \cup C_{2}\right) \cup\left(L_{1} \cup \cdots \cup L_{m}\right)\right) \cap X=X \backslash\{P\}$. By taking $F=\left(C_{1} \cup C_{2}\right) \cup$ $\left(L_{1} \cup \cdots \cup L_{m}\right)$, we have $\operatorname{deg}(F)=4+m=\lceil(d-1) / 2\rceil-1$, which yields the assertion.

Now we will show that there are conics $C_{1}$ and $C_{2}$ satisfying the above condition. First let us take a conic $C_{1}$ with $P \notin C_{1}$ containing at least 5 points of $X$, since $X$ is not in a conic. If $C_{1}$ contains more than 5 points of $X$, then we can easily take $C_{2}$ with $P \notin C_{2}$ such that $C_{2}$ contains at least 4 points of $X$. Thus we are done. So we may assume that $C_{1}$ contains exactly 5 points of $X$. Or, if there is another conic $C_{2}$ with $P \notin C_{2}$ which contains at least 5 points of $X \backslash\left(X \cap C_{1}\right)$, then the assertion is proved.

Now we have to consider the case that $X \cap C_{1}$ consists of exactly 5 points and the conics containing $X \backslash\left(X \cap C_{1}\right)$ always contain the point $P$. So let us take a conic $C^{\prime}$ with $P \in C^{\prime}$ containing at least 5 points of $X \backslash\left(X \cap C_{1}\right)$. Then we will show that the conic $C^{\prime}$ contains all the points of $X \backslash\left(X \cap C_{1}\right)$. If not, there is a point $P^{\prime} \in X \backslash\left(X \cap C_{1}\right)$ is not contained in $C^{\prime}$. Then the points $P^{\prime}$ and 4 fixed points $Q_{1}, \ldots, Q_{4}$ of $\left(X \cap C^{\prime}\right) \backslash\left(\left(X \cap C_{1}\right) \cup\{P\}\right)$ give a conic $C^{\prime \prime}$ containing these points. Then the conic $C^{\prime \prime}$ always contains $P$ from the assumption. Since the conics $C^{\prime}$ and $C^{\prime \prime}$ contains the 5 points $P, Q_{1}, \ldots, Q_{4}$ not in a line, we see $C^{\prime}=C^{\prime \prime}$, which contradicts $P^{\prime} \notin C^{\prime}$. Thus we have that the conics $C_{1}$ and $C^{\prime}$ cover $X$.

Now let $t=\operatorname{card}\left(X \backslash\left(X \cap C^{\prime}\right)\right)$. Note that $2 \leq t \leq 5$, since $t=1$ yields the case (ii) in (3.1). Let us take a point $P_{1}$ with $P_{1} \notin C^{\prime}$. The conic $C^{\prime \prime}$ through $P$ and $\left(X \cap C_{1}\right) \backslash\left\{P_{1}\right\}$ does not contain $X \cap C^{\prime}$. In fact, $X \cap C^{\prime} \subseteq C^{\prime \prime}$ gives $X \backslash$ $\left\{P_{1}\right\} \subseteq C^{\prime}$, which contradicts $t \geq 2$. So there is a point $P_{2}$ of $X \cap C^{\prime}$ such that $P_{2} \notin C^{\prime \prime}$. Then the conic $C_{1}^{\prime}$ through $P_{2}$ and $\left(X \cap C_{1}\right) \backslash\left\{P_{1}\right\}$ does not contain $P$, since $P \in C_{1}^{\prime}$ gives $C_{1}^{\prime}=C^{\prime}$. Let us fix 4 points $P_{3}, \ldots, P_{6}$ from $\left(X \cap C^{\prime}\right) \backslash$ $\left\{P, P_{2}\right\}$. Then the conic $C_{2}^{\prime}$ through $P_{1}, P_{3}, P_{4}, P_{5}, P_{6}$ does not contain $P$, since $P \in C_{2}^{\prime}$ gives $C_{2}^{\prime}=C^{\prime}$. Thus we see that $C_{1}^{\prime} \cup C_{2}^{\prime}$ contains at least 10 points with $P \notin C_{1}^{\prime} \cup C_{2}^{\prime}$. Hence the assertion is proved.

Acknowledgement. We are grateful to the referee for his critical remarks which lead to an improvement of the presentation of the paper. We also would like to thank Professor Kohji Yanagawa for his advice.

\section{REFERENCES}

[ 1 ] E. Arbarello, M. Cornalba, P. A. Griffiths and J. Harris, Geometry of algebraic curves I, Grundlehren der Math. Wissenschaften 167, Springer, 1985. 
[2] E. Ballico, On singular curves in positive characteristic, Math. Nachr. 141 (1989), 267-273.

[ 3 ] E. Ballico, On the general hyperplane section of a curve in char. $p$, Rend. Istit. Mat. Univ. Trieste 22 (1990), 117-125.

[4] E. Ballico AND C. MiYazaki, Generic hyperplane section of curves and an application to regularity bounds in positive characteristic, J. Pure Appl. Algebra 155 (2001), 93-103.

[5] E. Ballico and K. Yanagawa, On the $h$-vector of a Cohen-Macaulay domain in positive characteristic, Comm. Algebra 26 (1998), 1745-1756.

[6] A. Geramita and J. Migliore, Hyperplane sections of a smooth curve in $\mathbf{P}^{3}$, Comm. Algebra 17 (1989), 3129-3164.

[ 7 ] M. Kreuzer, On the canonical module of a 0-dimensional scheme, Can. J. Math. 46 (1994), 357-379.

[ 8 ] P. Maroscia, Some problems and results on finite sets of points in $\mathbf{P}^{n}$, Algebraic geometryopen problems, Lecture notes in math. 997, Springer, 1983, 290-314.

[9] C. Miyazaki, Sharp bounds on Castelnuovo-Mumford regularity, Trans. Amer. Math. Soc. 352 (2000), 1675-1686.

[10] J. Rathmann, The uniform position principle for curves in characteristic p, Math. Ann. 276 (1987), 565-579.

Department of Mathematical Sciences

UNIVERSITY OF THE RYUKYUS

NisHIHARA-CHO

OKINAWA 903-0213

JAPAN

E-mail: miyazaki@math.u-ryukyu.ac.jp 